\title{
Le vieillissement
}

\section{Une histoire de dommages de I'ADN, d'enveloppe nucléaire altérée et d'inflammation?}

> Le vieillissement est associé à une accumulation de cellules sénescentes produisant un environnement cellulaire inflammatoire qui pourrait expliquer différentes maladies liées à l'âge. Diverses situations menant à la sénescence sont liées à la présence de dommages de l'ADN. De plus, de nombreux syndromes progéroïdes sont associés à une instabilité du génome ou de la structure nucléaire. Nous discuterons du lien étroit existant entre l'altération des lamines, composants de l'enveloppe nucléaire, et le vieillissement cellulaire. Nous verrons que l'altération de l'enveloppe nucléaire, comme celle observée dans la Progéria, est aussi associée à des défauts de réparation de I'ADN, à une persistance de dommages de l'ADN et à un phénotype inflammatoire. <

Le vieillissement s'accompagne d'un nombre croissant de cellules sénescentes dans l'organisme. Ce processus participe à l'accumulation d'altérations fonctionnelles dans les tissus, menant à l'apparition de multiples maladies liées à l'âge [1]. Le phénomène de sénescence cellulaire a été décrit par Hayflick et Moorhead en 1961 comme étant un arrêt de la division cellulaire normale. Ces auteurs mirent en effet en évidence le caractère limité de la capacité mitotique des cellules normales humaines, introduisant ainsi le concept de sénescence réplicative. Depuis ces premières observations, la caractérisation de ce phénotype et de ses mécanismes d'induction par divers stress cellulaires a été largement précisée et nourrie par de nouvelles connaissances. La majorité des syndromes de vieillissement accéléré sont des maladies majoritairement associées à un défaut de signalisation ou de réparation des lésions à I'ADN ou à des défauts de structure de l'enveloppe nucléaire (Figure IA). Nous verrons qu'une caractéristique récurrente d'un grand nombre de situations d'induction de sénescence est la présence de dommages de I'ADN. En outre, la découverte du processus

Vignette (Photo @ Inserm - Foray Nicolas)
Paula Fontanilla*, Simon Willaume*, Benoit Thézé*,
Angela Moussa, Gaëlle Pennarun, Pascale Bertrand

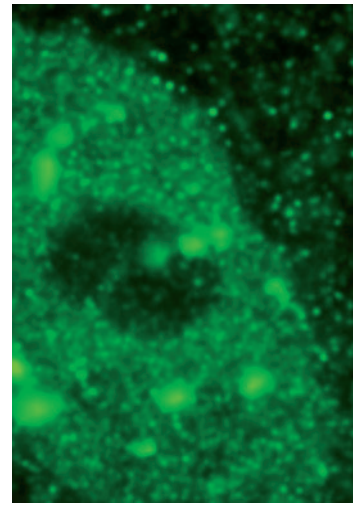

Laboratoire Réparation et Vieillissement, Institut de radiobiologie cellulaire et moléculaire, Institut de biologie François Jacob, Direction de la recherche fondamentale du CEA, Unité 1274, Stabilité génétique, cellules souches et radiations CEA-Inserm-Universités Paris Diderot-Paris Saclay, 18 route du Panorama, 92265 Fontenay-aux-Roses, France.

pascale.bertrand@cea.fr *Contribution équivalente

de sécrétion pro-inflammatoire des cellules sénescentes a permis d'établir un lien clair entre sénescence et vieillissement au niveau de l'organisme entier et d'appréhender les liens communs des maladies liées à l'âge.

Nous décrirons dans cette revue les caractéristiques et les mécanismes d'induction de la sénescence, ainsi que le phénotype sécrétoire et inflammatoire qui lui est associé. Nous discuterons du lien étroit existant entre l'altération des lamines, composants essentiels de l'enveloppe nucléaire et le vieillissement cellulaire. Nous verrons que l'altération de l'enveloppe nucléaire, comme celle observée dans la Progéria, est aussi associée à des défauts de réparation de l'ADN, à une persistance accrue de dommages de l'ADN et à un phénotype inflammatoire.

\section{Caractéristiques des cellules sénescentes et rôle dans le vieillissement de l'organisme}

L'existence de plusieurs programmes d'induction de la sénescence, selon le type cellulaire et les stimulus de stress subis, mène à une complexité du phénotype sénescent. Encore actuellement, les marqueurs permettant sa détection restent largement non spécifiques et ce n'est que l'observation conjointe de plusieurs caractéristiques associées à ce phénotype qui permet de conclure à un état de sénescence. Ainsi, la cellule sénescente est généralement caractérisée par une activation persistante de la réponse aux dommages de l'ADN. Cette dernière peut être mise en évidence par l'accumulation de cicatrices non résolues au niveau de I'ADN (DNA scars) révélées par les marqueurs de cassures d'ADN double-brin (CDB) sous forme de foyers de réparation $\gamma-\mathrm{H} 2 \mathrm{AX}$ et $53 \mathrm{BPl}$ [2]. Toutefois, la persistance de dommages n'est pas spécifique de la sénescence. Cette 


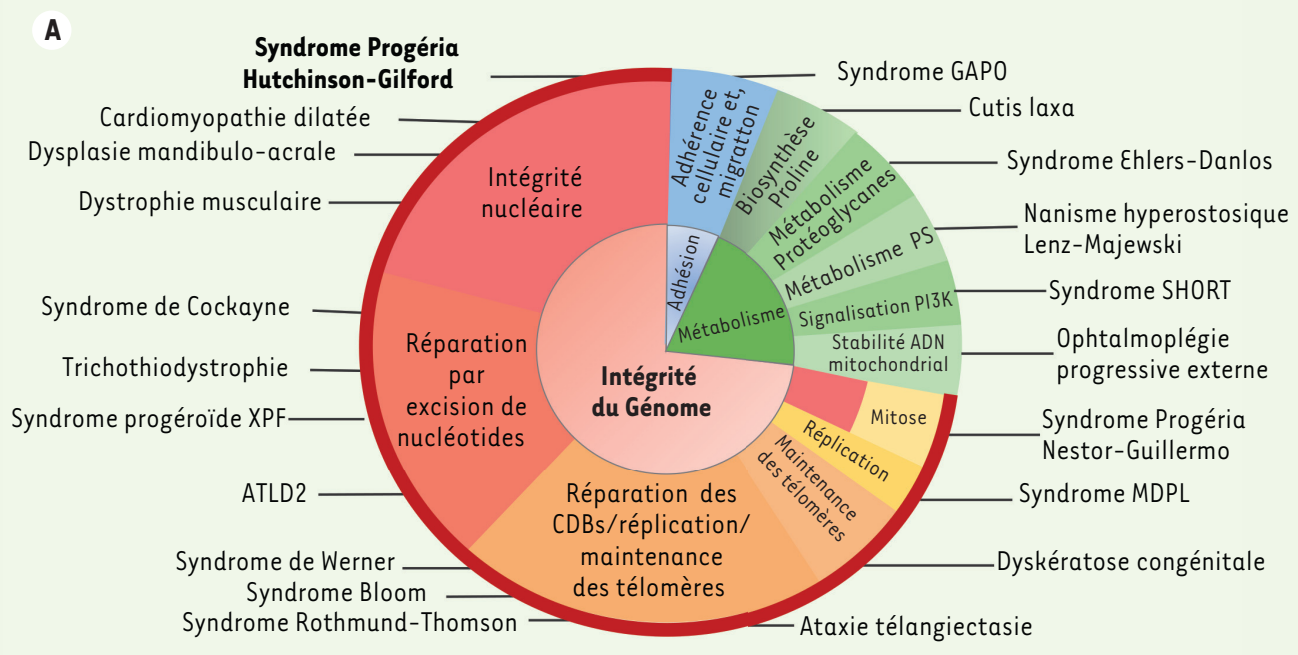

B

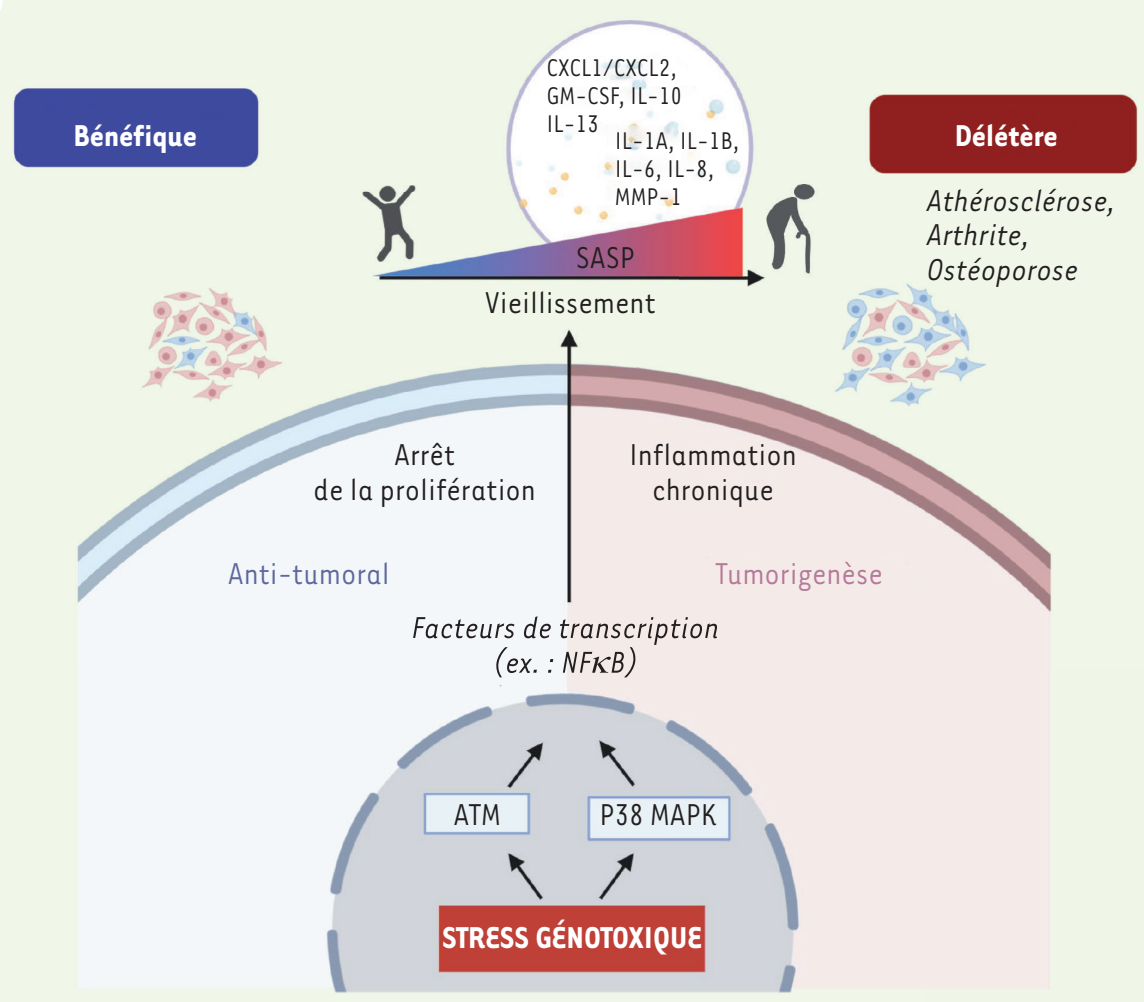

Figure 1. A. Pathologies de vieillissement accéléré. Une majorité des syndromes progéroïdes est associée à des mutations génétiques menant à une instabilité chromosomique (gènes impliqués dans la réparation de l’ADN, la réplication, la mitose ou la stabilité télomérique, etc.) ou à une altération de l'intégrité de la structure nucléaire (lamines, etc.) (modifiée d'après [62]). B. La sénescence et le SASP : des armes à double tranchant. Au cours de la vie, la sénescence aurait un rôle bénéfique et même anti-tumoral en bloquant la prolifération de cellules porteuses de dommages, et en recrutant des cellules immunitaires pour éliminer ces cellules « anormales ». Cependant au cours du vieillissement normal, l'accumulation de cellules sénescentes (visualisées ici par des cellules bleues), associée à l'affaiblissement de la réparation de l'ADN et du système immunitaire engendrerait une inflammation chronique, liée au phénotype sécrétoire associé à la sénescence (SASP). Ceci pourrait rendre compte de nombreuses maladies liées à l'âge, dont l'athérosclérose, l'arthrite, l'ostéoporose et le cancer. Cette opposition fonctionnelle de la sénescence au cours de la vie de l'organisme est en accord avec le concept de pléiotropie antagoniste. La persistance des dommages et les voies de signalisation NF- $\kappa B$ et $p 38$ MAPK sont impliquées dans l'induction du SASP. 
dernière se caractérise principalement par le blocage persistant du cycle cellulaire, initié par la réponse aux dommages de l'ADN et contrôlé par la modulation d'inhibiteurs de kinases dépendantes des cyclines (CDKi). En particulier, la régulation des CDKi p16 ${ }^{\text {INK4a }}$ et p2 $1^{\text {Cipl }}$, l'activation des gènes suppresseurs de tumeurs $\mathrm{p} 53$ et $\mathrm{pRb}$ (retinoblastoma protein), le contrôle des voies mTOR (mechanistic target of rapamycin) et p38 MAPK (mitogen-activated protein kinase) participent à la mise en place du programme de sénescence [3] $(\rightarrow)$.

$\rightarrow$ Voir la Synthèse de J.M. Brondello et al., $m / s n^{\circ} 3$, mars 2012, page 288

L'analyse de l'expression de ces protéines, mais aussi l'incapacité de la cellule à incorporer les analogues de bases $\mathrm{BrdU} / \varepsilon \mathrm{dU}^{1}$ au niveau de I'ADN, permet de déterminer l'arrêt «permanent » du cycle cellulaire associé à l'état de sénescence. Les cellules sénescentes développent également des capacités de résistance à l'apoptose, notamment en augmentant la quantité de protéines anti-apoptotique $\mathrm{Bcl}(B$-cell lymphoma)-2, Bcl-w et Bcl-xL [4]. Cependant, leur niveau d'expression a rarement été utilisé comme marqueur.

Par ailleurs, la cellule en sénescence est caractérisée par une sécrétion accrue (nommée SASP pour senescence-associated secretory phenotype) de facteurs inflammatoires et de remodelage tissulaire, tels que des cytokines, des chimiokines et des protéinases. Ce profil sécrétoire est très hétérogène et régulé par le facteur de transcription $N F-\kappa B$ (nuclear factor-kappa $B$ ) et la kinase p38 MAPK, eux-mêmes contrôlés par la réponse aux dommages à I'ADN, ainsi que par la voie de détection d'ADN dans le cytoplasme des cellules via le senseur cGAS. Les composants principaux du SASP (IL[interleukine]-1 $\alpha, I L-1 \beta, I L-6$, IL-8, MMP-1 [matrix metalloproteinase-1]) sont pro-inflammatoires, bien que d'autres médiateurs exprimés soient immunosuppresseurs (CXCL[C-X-C motif chemokine ligand] 1 et 2, GM-CSF [granulocyte macrophage colony-stimulating factor], M-CSF, IL-10, IL-13) [5]. La constitution du SASP est par ailleurs soumise à un contrôle spatial et temporel [6]. Le SASP confère à la sénescence un rôle parfois bénéfique dans la réparation et le remodelage tissulaire et parfois délétère dans de nombreuses pathologies inflammatoires et dans le développement du cancer. Il est ainsi proposé que la sénescence puisse avoir un rôle anti-tumoral, d'une part en bloquant la prolifération de cellules portant des dommages de l'ADN, potentiellement tumorigènes, et, d'autre part, en déclenchant l'immunité innée contre celles-ci. Cependant, au cours du vieillissement normal, l'accumulation de cellules sénescentes, associée à l'affaiblissement de la réparation de l'ADN et du système immunitaire engendrerait une inflammation chronique liée au SASP, qui pourrait rendre compte de la plupart des pathologies liées à l'âge, dont l'athérosclérose, l'arthrite, l'ostéoporose et le cancer (Figure IB). Cette opposition fonctionnelle de la sénescence au cours de la vie de l'organisme est en accord avec l'hypothèse de pléiotropie antagoniste décrite par George C. Williams en 1957 [7]. Le lien direct entre accumulation de cellules sénescentes et vieillissement de l'organisme a été proposé ou discuté par différentes équipes [3, 8]. Enfin, plus récemment, les travaux de Baker et al. ont apporté la preuve de concept dans un modèle murin que l'élimination des cellules

${ }^{1}$ 5-bromo-2'-deoxyuridine/5-éthynyl-2'-deoxyuridine sénescentes induisait une diminution de différentes pathologies liées à l'âge [9]. Ces travaux justifient les recherches actives actuellement menées sur les mécanismes moléculaires associés à la sénescence et celles ayant pour but de découvrir des molécules sénolytiques, qui permettraient d'éliminer les cellules sénescentes au niveau de l'organisme [10].

Ces dernières années, il a été montré que les cellules sénescentes sont la cible de l'immuno-surveillance dans différents contextes physiologiques. Par ce mécanisme, le système immunitaire détecte et élimine les cellules sénescentes ainsi que les cellules (pré-) malignes, réduisant ainsi le risque de développer un cancer. La dérégulation de ce processus contribue à l'accumulation de cellules sénescentes dans l'organisme vieillissant. Récemment, il a été montré la perforine-l joue un rôle essentiel pour limiter la charge tissulaire en cellules sénescentes tout au long du processus de vieillissement. Ainsi, une carence en perforine-1 est accompagnée d'une inflammation chronique et d'un vieillissement accéléré. La déficience en perforine-1 dans un modèle murin de la Progéria $\left(\operatorname{LMNA}^{+/ 6609 G}\right)^{2}$, accélère le vieillissement prématuré et, remarquablement, le traitement par le sénolytique ABT-737 (mimétique BH3) réduit l'impact du vieillissement prématuré dans ce modèle [11]. Quelles sont les cibles reconnues par les cellules immunitaires exprimant la perforine-1 ?, Quel est le mécanisme exact d'action du sénolytique ABT737 dans le modèle Progéria? Autant de questions qui nécessitent des recherches actives dans ce domaine de l'immunosurveillance [12]. L'immunosurveillance de la sénescence apparaît donc comme un facteur limitant du vieillissement ainsi que du développement tumoral. Les cellules sénescentes présentent des modifications métaboliques, des aberrations structurelles et morphologiques (élargissement de la cellule), des changements de la composition de la membrane plasmique et une accumulation de mitochondries ainsi que de lysosomes. Cette augmentation d'activité lysosomale n'est pas nécessaire à l'induction de la sénescence, mais est facilement décelable par la quantification de la SA-bêta-galactosidase, un test classiquement utilisé pour mettre en évidence un phénotype sénescent. Des changements importants de l'organisation nucléaire sont également observés au cours de la sénescence, caractérisés par la présence de foyers d'hétérochromatine (SAHF) et, comme nous le verrons, par la dérégulation de la lamina nucléaire.

\footnotetext{
${ }^{2}$ Gène codant la lamine A.
} 


\section{Mécanismes d'induction de la sénescence : le rôle pivot des dommages de l'ADN}

\section{Sénescence réplicative : rôle des télomères}

La sénescence réplicative, observée par Hayflick en 1961, est liée au problème de réplication au niveau des extrémités des chromosomes. En effet, les ADN polymérases synthétisent l'ADN dans la direction 5' vers 3 ' et nécessitent une amorce afin de générer un brin néoformé à partir de la séquence de référence. Au niveau des extrémités télomériques, à chaque cycle réplicatif de la cellule, la partie terminale du brin dit retard ne pourra donc pas être copiée par l'ADN polymérase. II en résulte un raccourcissement progressif des télomères à chaque cycle de division cellulaire. À partir d'un certain degré de raccourcissement des chromosomes, le complexe multi-protéiques appelé «Shelterin» peine alors à se former autour des télomères et la réponse aux dommages à I'ADN s'en trouve alors durablement activée. Ce complexe est en effet capital pour former la structure en boucle $T$ ( $T$-loop) des télomères et aussi pour empêcher les protéines de la réponse aux dommages de l'ADN de reconnaître l'extrémité des télomères comme une cassure double-brin. Le raccourcissement télomérique conduit à un arrêt du cycle cellulaire, à l'activation de mécanismes de réparation, mais aussi au déclenchement de la sénescence et des voies de mort cellulaire.

\section{Sénescence induite par l'expression d'oncogènes}

D'autres stimulus peuvent être responsables de l'induction de la sénescence, comme cela fut initialement montré par les travaux de Scott Lowe [13]. En effet, une activation chronique d'oncogènes (Ras, $B R A F$, etc.) ou encore l'inactivation de protéines suppresseurs de tumeurs (PTEN, RB, etc.) peuvent induire l'entrée en sénescence. La sénescence induite par l'expression d'oncogènes (OIS) a été décrite dans un premier temps comme ayant un rôle anti-tumoral.

L'OIS peut notamment être causée par des variants constitutivement actifs de la voie RAS/MAPK (H-RAS ${ }^{G 12 V}, N-R^{6 S} S^{061 R}, B-R A F^{V 600 \varepsilon}$ ), ainsi que par des variants de la voie PI3K (phosphoinositide 3-kinase)/AKT (protéine kinase $B$ ) (PIK3CA ${ }^{H 1047 R}, A K T 1^{\varepsilon 17 K}$, délétions ou mutations de PTEN) [14]. Dans le cas de la RIS (RAS-induced senescence), l'hyperprolifération engendrée par la dérégulation de ces voies de signalisation contribue à générer un stress réplicatif (associé potentiellement à l'induction d'espèces réactives de l'oxygène) et à activer la réponse aux dommages de l'ADN [15]. II en résulte l'activation des protéines ATM/ATR (ataxia telangiectasia mutated/ataxia telangiectasia and Rad3-related protein), la surexpression des protéines $p 21^{\text {Cipl }}$ et $p 16^{\text {INK4a }}$ (dont dépend le maintien de ce phénotype sénescent), et l'inhibition de la phosphorylation de $\mathrm{pRb}$. L'activation des protéines $p 21^{\text {Cipl }}$ et p16 $6^{\text {INK4a }}$ réprime respectivement les complexes CDKl/2-cyclines $A / \varepsilon$ et CDK4/6-cycline D. Cela permet l'activation de la protéine $\mathrm{pRb}$ dont la forme non phosphorylée séquestre les facteurs de transcription de la famille $\varepsilon 2 \mathrm{~F}$ et réprime les gènes contrôlant la division cellulaire. Or dans de nombreux cancers, les kinases de contrôle du cycle (telles que la cycline $\varepsilon$ ) et $\mathrm{Rb}$ sont fréquemment dérégulées. Cela ouvre un rationnel pour le développement de thérapies pro-sénescence, qui pourraient constituer une approche efficace dans le traitement de nombreux cancers [10].

En dépit de ce schéma général, selon l'oncogène exprimé ou le modèle cellulaire utilisé, la sénescence peut-être induite sans implication de la réponse aux dommages de I'ADN ou de l'attrition des télomères $[16,17]$. À titre d'exemple, Jeanblanc et al. ont montré qu'une sénescence induite par C-RAF pouvait s'établir malgré l'inhibition de la signalisation des dommages à I'ADN, suggérant ainsi l'existence d'une voie de déclenchement de la RIS indépendante de la DDR [18]. De même, Michaloglou et al. ont souligné les différences de réponses cellulaires et de sénescence, suite à l'expression de $B-R^{2} F^{V 600 \varepsilon}$, selon le type de tumeurs bénignes et malignes [19]. De plus, la sénescence induite par la voie AKT est dépendante de mTOR et ne semble pas nécessiter de réponse aux dommages à l'ADN et dans ce cas, la protéine $\mathrm{pl} 6^{\mathrm{INK} 4 \mathrm{a}}$ reste à des niveaux modestes. $\varepsilon n$ effet, l'hyperactivation d'AKT induit la sénescence en activant la synthèse de p53 via mTORCl [20]. D'autres travaux proposent que l'interaction entre MDM2 (murine double minute 2) et $p 53$ serait inhibée (par séquestration de MDM2 dans un complexe comportant des protéines nucléolaires) (pour revue voir [14]).

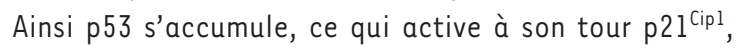
dont dépend l'arrêt prolongé du cycle cellulaire. De nombreux autres acteurs à potentiel oncogénique (tels que c-Myc) peuvent être impliqués dans l'ols et les études sur ce type de sénescence présentent un certain nombre de particularités selon le variant d'oncogène étudié et le modèle cellulaire utilisé [17].

Malgré tout, différentes situations de sénescence induite par des oncogènes sont associées à la persistance de dommages qui participent directement ou indirectement à la mise en place de la sénescence.

\section{Le stress oxydant}

Des agents oxydants (tels que l' $\mathrm{H}_{2} \mathrm{O}_{2}$, ou les espèces réactives de l'oxygène générées par le métabolisme cellulaire, ROS) sont capables d'induire la sénescence, comme cela a été suggéré par Denham Hartman voici plus de 60 ans dans sa «théorie radicalaire du vieillissement ». Ce stress oxydant va notamment induire des lésions à I'ADN (en plus de l'oxydation des protéines) et potentiellement, selon le dommage oxydatif, un blocage ou un ralentissement des fourches de réplication. Une anomalie de fonctionnement des mitochondries génère une augmentation de radicaux libres dans la cellule et peut induire une sénescence associée à la dysfonction mitochondriale [21]. Des voies de signalisation vont également pouvoir être activées, telles que p38 MAPK [22] ou NRF2 (nuclear factor erythroid- 


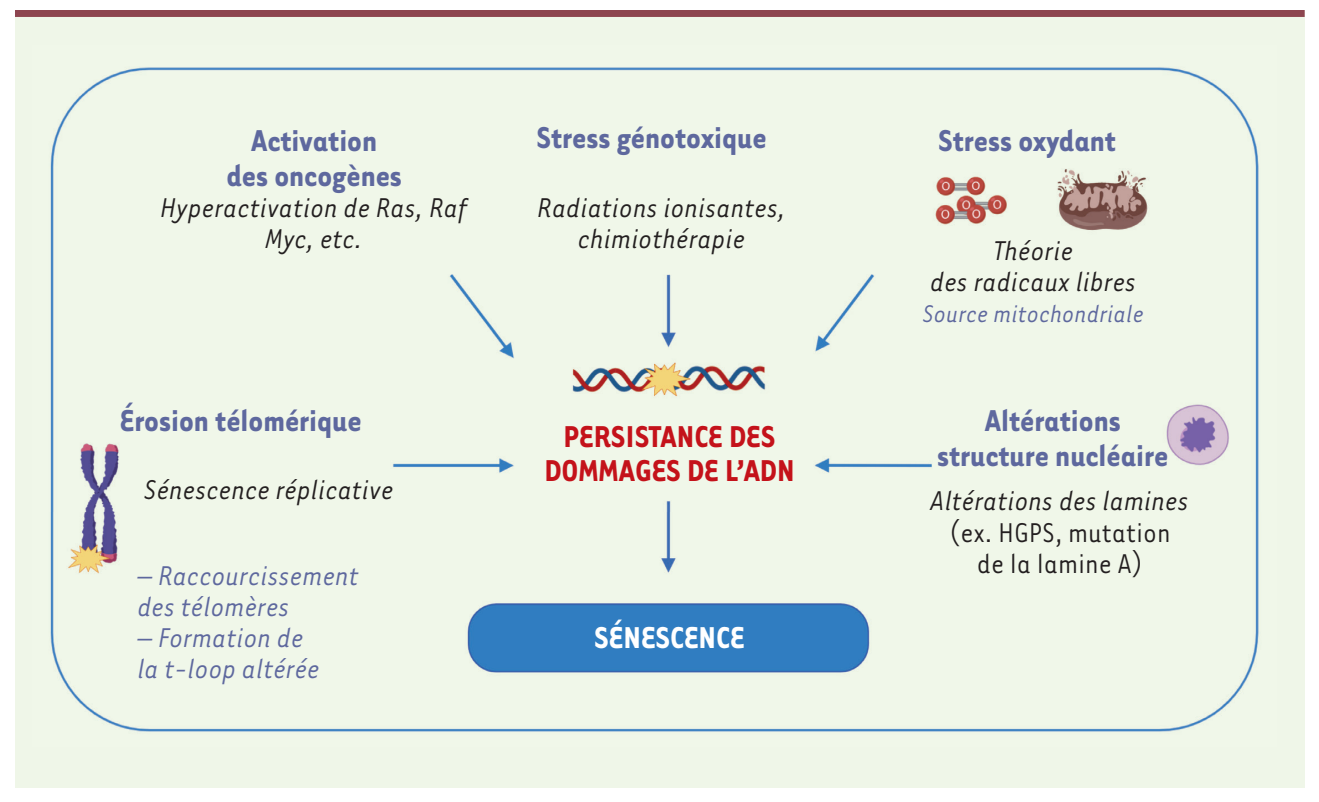

Figure 2. Des dommages de l'ADN : un élément commun entre différentes situations de sénescence. Différentes situations menant à l'induction de la sénescence sont associées à l'accumulation de dommages de I'ADN : érosion télomérique (sénescence réplicative), hyperactivation d'oncogènes (OIS), exposition (thérapeutique ou accidentelle) à des agents génotoxiques, stress oxydant et altérations de la structure nucléaire (altération du niveau des lamines, comme dans la Progéria.

2-related factor 2) [23]. L'ensemble de ces observations place ainsi la sénescence au cœur des théories sur les liens entre radicaux libres et vieillissement cellulaire.

\section{Les agents génotoxiques}

Selon leurs mécanismes d'actions, de nombreux agents génotoxiques, tels que les radiations ionisantes ou les molécules chimio-thérapeutiques, vont générer des dommages à l'ADN, une perturbation du cycle cellulaire ou un stress oxydant, et donc potentiellement induire la sénescence. Les inhibiteurs de méthylases de l'ADN ou de déacétylases d'histones peuvent également déclencher la sénescence, en perturbant l'organisation chromatinienne et en déréprimant le suppresseur de tumeur pl6 $6^{\text {INK4a }}[24]$.

\section{Induction paracrine par les médiateurs du SASP}

Une dernière forme d'induction de la sénescence est paracrine. Le SASP d'une cellule primaire en sénescence va en effet être capable d'induire la sénescence au niveau de cellules secondaires voisines. La sénescence paracrine va ainsi favoriser l'amplification du phénotype [25]. Le SASP, dont le mécanisme sera précisé ci-dessous, est lui aussi associé à la présence ou à la persistance de dommages [26].

Au sein des divers programmes de sénescence que nous avons mentionnés (attrition des télomères, OIS, stress oxydant, etc.), la réponse aux dommages de l'ADN est souvent une étape centrale dans le contrôle de l'entrée en sénescence (Figure 2). La présence de cassures doubles brins non résolues au niveau de l'ADN induit en effet le recrutement de la kinase ATM dont l'activation permet la phosphorylation des histones H2AX autour de la cassure ainsi que l'induction de nombreux effecteurs, parmi lesquels, les kinases $\mathrm{CHK}$ l et $\mathrm{CHK} 2$, et p53, qui a leur tour activent l'expression de nombreux gènes, notamment ceux permettant la mise en place de la sénescence lorsque l'activation de ces voies de signalisation persiste durablement.

\section{Dommages à I'ADN, ADN cytosoliques, SASP et inflammation}

L'un des éléments clés du vieillissement est la sécrétion par les cellules sénescentes de cytokines inflammatoires, facteurs de croissance et autres protéines. Comme nous l'avons décrit précédemment, le SASP a un rôle majeur dans de nombreux désordres associés à la sénescence. Le lien moléculaire entre dommages de I'ADN et ce phénotype pro-inflammatoire a été récemment éclairci. Dans la cellule, l'inflammation est majoritairement initiée via des récepteurs du système immunitaire inné, tels que les nombreux PRR (pattern recognition receptors) permettant d'identifier des pathogènes. Un senseur de l'ADN, comme le cGAS est capable de détecter de l'ADN présent dans le cytosol, de produire du c-GAMP et d'activer la protéine STING. Cette dernière phosphoryle TBKl (TANK binding kinase 1) qui à son tour, induit la phosphorylation du facteur de transcription IRF3 (interferon regulatory factor 3) permettant l'activation des gènes codant les interférons de type $l$ et la réponse immunitaire innée. Lors de dommages à l'ADN, la présence de fragments d'ADN augmente dans le cytoplasme (probablement produits par les mécanismes de réparation de l'ADN ou le blocage de fourches de réplication) et déclenche une réponse inflammatoire innée via la voie cGAS-STING [27-29] $(\rightarrow)$.

$(\rightarrow)$ Voir la Synthèse de R. Chanut et $V$. Petrilli, $m / s n^{\circ}$ 6-7, juin-juillet 2019, page 527

Dans le cas des cellules sénescentes, il est possible d'observer la présence de fragments de chromatine cytoplasmique (CCF) [30]. cGAS se localise alors avec 


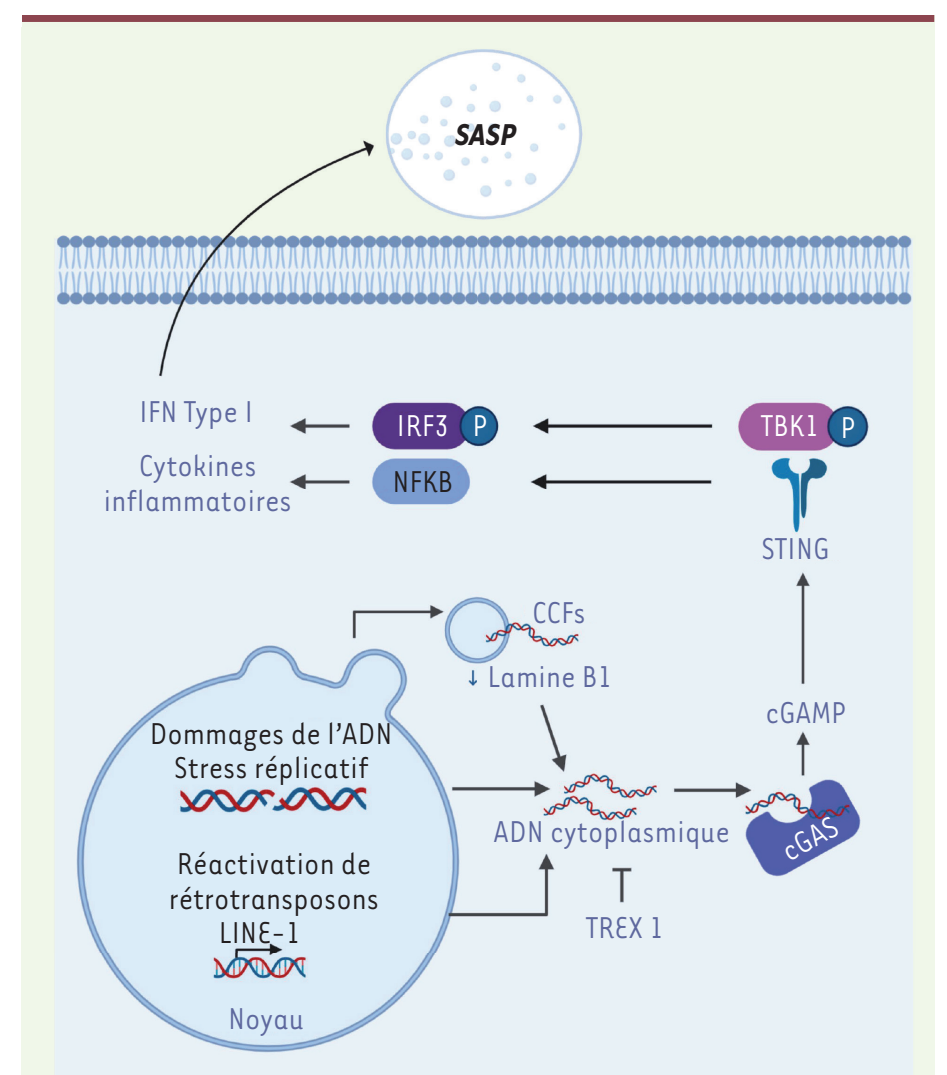

Cytoplasme

Figure 3. Rôle de cGAS dans la sénescence et l'inflammation associée. Récemment, il a été montré que la voie cGAS-STING était impliquée dans l'induction du SASP. En cas de dommages de I'ADN (ou réactivation de transposon), des fragments d'ADN s'acccumulent dans le cytosplasme (notamment dans des micro-noyaux). Ces ADN cytosoliques sont reconnus par le senseur cGAS, qui en produisant un dinucléotide cyclique c-GAMP, va activer STING et ainsi induire la production d'interféron de type I, via la phosphorylation de TBKI et d'IRF3, ainsi que la sécrétion de cytokines inflammatoires, via l'activation de la voie NF- $\kappa B$. La diminution de la lamine Bl observée au cours de la sénescence peut aboutir à une fragilité de l'enveloppe des micro-noyaux, favorisant ainsi l'accès des fragments de chromatine (CCF) à cGAS.

les CCF, ce qui mène à la production de cytokines inflammatoires et participe ainsi au SASP (Figure 3). La délétion de cGAS inhibe la sénescence induite par un stress oxydant [31] ou par des radiations [30]. L'ensemble de ces données démontrent que cGAS est essentiel à la production du SASP et à l'induction de la sénescence cellulaire elle-même [32]. Une réactivation de rétrotransposons de type LINE-1 a également été mise en évidence lors de la sénescence. Ces éléments mobiles sont aussi l'une des sources d'ADN cytosoliques participant à la mise en place du SASP [33] et au micro-environnement pro-inflammatoire des cellules sénescentes.

Le déclenchement de l'immunité innée, permet le recrutement de cellules immunitaires pour détruire les cellules sénescentes potentiellement porteuses de dommages de l'ADN. Ce phénomène pourrait donc être considéré comme anti-tumoral. Cependant un environnement inflammatoire peut aussi accroître l'agressivité de cellules tumorales par le déclenchement, par exemple, d'une transition épithélio-mésenchymateuse (EMT). L'accumulation de cellules sénescentes au cours du vieillissement normal de l'organisme engendre donc une inflammation chronique et serait à l'origine de nombreuses pathologies liées à l'âge (canitie, athérosclérose, ostéoporose, cataracte, cancer, etc.).

\section{Lamines et sénescence}

Un des syndromes de vieillissement prématuré les plus sévères, la Progéria, a été décrit par Jonathan Hutchison en 1886 et par Hasting Gilford en 1887 : il a été nommé syndrome Hutchison Gilford Progeria (HGPS). La mutation dans le gène de la lamine $A$, responsable de cette pathologie, a été identifiée en 2003 par les groupes de Francis Collins et de Nicolas Levy [34, 35]. Cette découverte a permis de mettre en évidence un lien entre les lamines, l'altération de l'enveloppe nucléaire et le vieillissement.

\section{Les lamines}

Les lamines sont des filaments intermédiaires de type $\mathrm{V}$, constituants essentiels de la lamina nucléaire, un réseau fibreux bordant la face interne de l'enveloppe nucléaire. La lamina joue un rôle prépondérant dans le maintien de la forme du noyau de la cellule, dans l'organisation du génome et ses fonctions. La famille des lamines est composée de plusieurs protéines: les lamines de types $A / C$ et les lamines de type $B$. Les lamines $A$ et $C$ codées par le même gène LMNA sont issues d'un épissage alternatif et sont présentes principalement dans les cellules différenciées. La lamine $B 1$ est codée par le gène $L M N B 1$. Les lamines $B 2$ et $B 3$ sont issues du même gène LMNB2 par épissage alternatif. Les lamines $B 1$ et $B 2$ sont présentes dans tous les types cellulaires. La lamine B3 est spécifique des cellules germinales. Il existe de nombreuses maladies, appelées laminopathies, associées à des dérégulations des lamines $A$ et $C$. L'une des plus emblématiques est la Progéria, une forme sévère de vieillissement accéléré.

\section{Dérégulation de la lamine $A$, cas des HGPS}

La Progéria est une maladie génétique très rare ( 1 cas pour 4 à 8 millions de naissances) dont la caractéristique principale est un vieillissement accéléré. Les enfants atteints développent les premiers symptômes pendant leurs premiers mois de vie (défaut de développement, peau avec une apparence de sclérodermie). 
Habituellement, autour de 18-24 mois après la naissance, il est possible d'observer une alopécie ainsi qu'une apparence caractéristique (petit visage avec une mâchoire en retrait, nez pincé) et un délai de sortie des dents primaires. Plus tard, d'autres symptômes apparaissent: sclérodermie, insuffisance rénale, diminution de la vision et problèmes cardiovasculaires. De plus, une dégénérescence musculosquelettique entraîne une perte de graisse corporelle et de muscles, des articulations raides, des dislocations de la hanche et d'autres symptômes généralement absents chez les personnes non âgées. Par contre, les individus conservent un développement mental et moteur normal, dû à l'expression d'un micro ARN (miR-9) qui inhibe l'expression de la prélamine $A$ dans le cerveau [36]. La majorité des patients meurent, durant l'adolescence, d'artériosclérose et de maladies cardiovasculaires. La Progéria est due à une mutation de la lamine A et à l'accumulation d'une forme immature de la protéine. En effet, la pré-lamine A codée par le gène LMNA subit une série de maturations post-transcriptionnelles, dont une carboxyméthylation suivie d'une farnésylation et d'un clivage des 15 derniers acides aminés par l'endoprotéase ZMPSTE24, éliminant ainsi la farnésylation dans la forme mature de la lamine $A$. La mutation la plus fréquente chez les patients atteints de HGPS est une mutation dans l'exon 11 de la lamine A. Cette mutation entraîne l'utilisation d'un site cryptique d'épissage des ARN messagers, aboutissant à la délétion de 50 acides aminés incluant le site de clivage par la ZMPSTE24. Cela conduit à la production d'une protéine immature mutée et farnésylée appelée progérine (Figure 4A). Au cours du vieillissement normal ou physiologique, les cellules expriment une forme immature de lamine $A$ [37], ce qui renforce l'idée que la lamine $A$ immature joue un rôle dans le vieillissement cellulaire, même physiologique.

\section{Lamine A, Progéria, réparation de I'ADN et stabilité télomérique}

$\varepsilon n$ plus des déformations de l'enveloppe nucléaire, les cellules déficientes en lamine A montrent des signes d'instabilité génétique, tels que des niveaux élevés de cassures d'ADN (révélées par le marqueur $\gamma$-H2AX), une augmentation des cassures chromosomiques, ainsi que des défauts au niveau des télomères. Cette instabilité serait en partie due à la déstabilisation d'une protéine clef du mécanisme de religature d'extrémités d'ADN non-homologues (NHEJ), la protéine 53BPl [38]. Une diminution d'expression de gènes codant des acteurs essentiels de la recombinaison homologue $(\mathrm{RH})$, autre mécanisme de réparation des cassures double-brin, a aussi été rapportée dans ces cellules [38]. Par ailleurs, l'expression de prélamine $A$ entraîne une dérégulation de la nucléoporine Nup153, menant à une mauvaise localisation de 53BPl et ainsi, à une diminution de l'efficacité de réparation [39]. En ce qui concerne les cellules de patients souffrant d'HGPS (cellules HGPS), l'accumulation de la progérine altère la forme du noyau des cellules, l'expression génique, la régulation épigénétique et cause une instabilité génétique générale. Ainsi, des fibroblastes de patients présentent une accumulation de dommages de I'ADN, une instabilité chromosomique, une sensibilité accrue aux agents génotoxiques, ainsi qu'une réponse aux dommages activée en permanence [40]. Par ailleurs, les cellules HGPS présenteraient un défaut de recrutement de RAD5l et BRCAl aux dommages, associé à une diminution d'expression de ces protéines. Ces dernières sont essentielles à la réparation des CDB par recombinaison homologue $[38,40]$. De plus, des données de la littérature suggèrent l'existence de liens étroits entre les lamines et le maintien des télomères. Ainsi, les cellules déficientes en lamine A ou exprimant la progérine présentent une altération de la localisation et de la mobilité des télomères, ainsi que des dommages télomériques et un raccourcissement de ces derniers [41]. Une interaction entre la lamine $A$ et la protéine du shelterin, TRF2, a été mise en évidence et jouerait un rôle dans la protection des télomères [42]. L'expression de la progérine induit la transcription d'ARN non-codants au niveau des télomères. Ces ARN participent à l'activation de la réponse aux dommages et seraient ainsi responsables de différents phénotypes télomériques, de la baisse de prolifération et de l'induction de sénescence des cellules HGPS [43]. L'activation de la réponse aux dommages de I'ADN aux télomères aurait donc un rôle majeur dans l'étiologie de la pathologie.

\section{Progéria, lamine A et stress oxydant}

L'une des sources endogènes d'instabilité génétique est le stress oxydant produit par le métabolisme des mitochondries. Les cellules HGPS présentent des phénotypes caractéristiques de dysfonctions mitochondriales, notamment une augmentation de la production d'espèces réactives de l'oxygène (ROS), ainsi qu'une sensibilité accrue à ces derniers. Des études ont montré qu'un traitement par la $\mathrm{N}$-acétyl-cystéine (NAC), qui diminue les ROS dans des cellules HGPS, provoquait une diminution du niveau basal de dommages de I'ADN ainsi qu'une augmentation de la prolifération cellulaire. Les ROS seraient donc un élément majeur contribuant à I'instabilité dans les cellules HGPS [44]. De plus, l'expression de la progérine altère la voie NRF2 de détoxification des ROS [45]. L'enveloppe nucléaire, comportant une concentration élevée d'enzymes de détoxification, pourrait servir de bouclier contre les ROS [46]. Ainsi, au cours de la sénescence, l'érosion ou la rupture de l'enveloppe nucléaire, induite par l'altération des lamines, pourrait perturber ce bouclier, modifier la détoxification des ROS et les échanges nucléo-cytoplasmiques (aboutissant à la translocation nucléaire de composants tels que des nucléases ou encore des mitochondries) et ainsi aboutir à l'augmentation du stress oxydant nucléaire, ainsi qu'à des dommages de I'ADN et à une instabilité génétique.

Progéria, lamine A, stress réplicatif et inflammation Les lamines jouent un rôle important dans les mécanismes de réplication de l'ADN. La lamine A stabilise 
A

\begin{tabular}{|c|c|}
\hline Maturation normale de la lamine $\mathbf{A}$ & Syndrome Progéria Hutchinson-Gilford (HGPS) \\
\hline 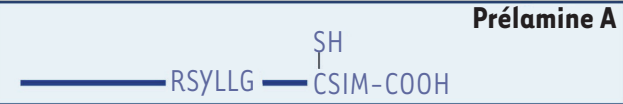 & 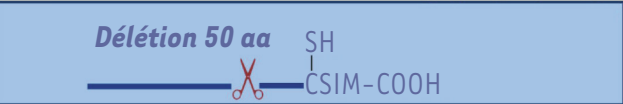 \\
\hline $\begin{array}{l}\text { Farnésylation } \\
\text { et carboxylation }\end{array}$ & $\downarrow \begin{array}{l}\text { Farnésylation } \\
\text { et carboxylation }\end{array}$ \\
\hline $\begin{array}{l}\text { Lamine A mature } \\
\text { (non farnésylée) } \\
\mathrm{RSY}+\mathrm{LLG}-\mathrm{C}-\mathrm{COCH} 3\end{array}$ & $\begin{array}{l}\text { Progérine } \\
\text { (farnésylée) }\end{array}$ \\
\hline
\end{tabular}

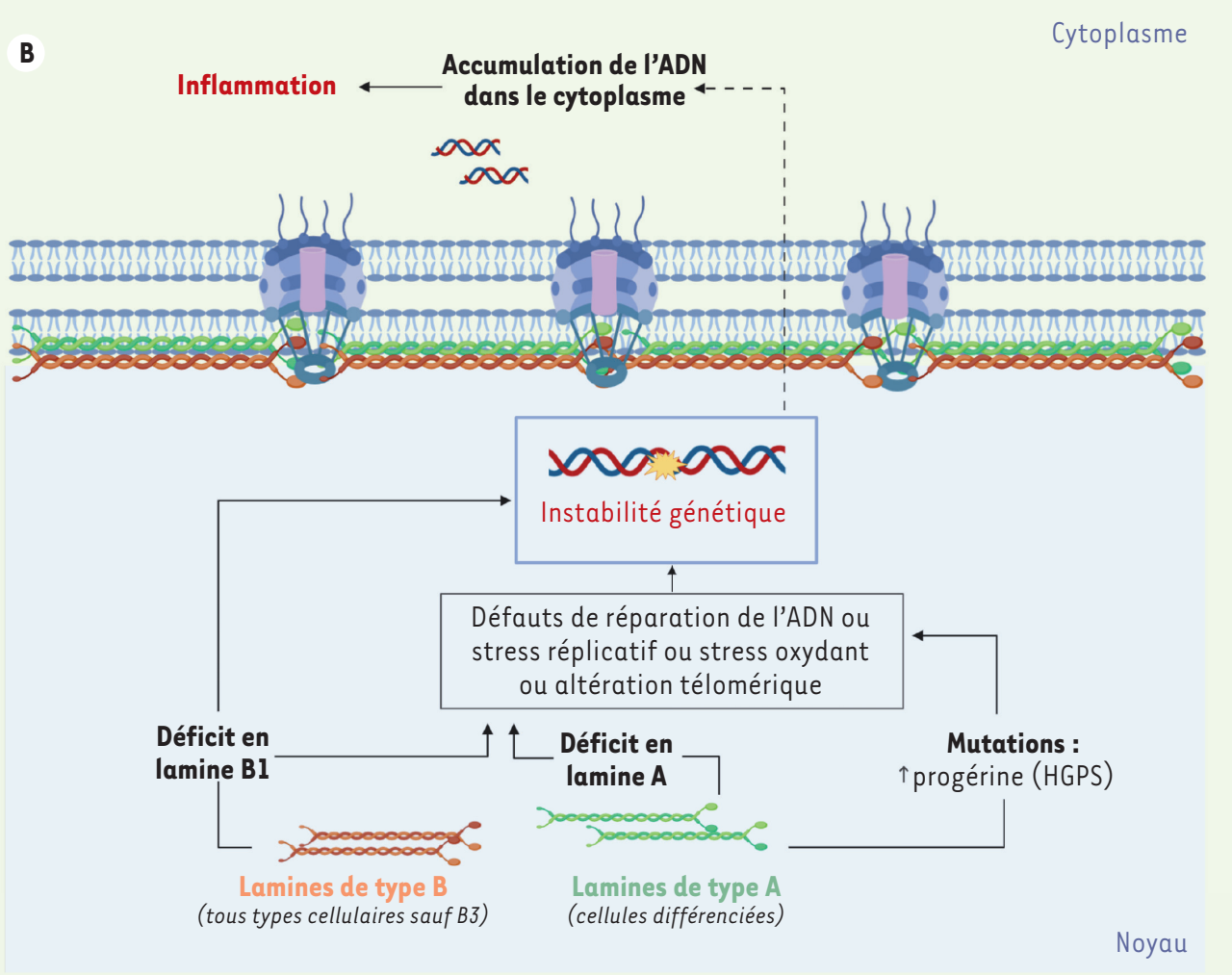

Figure 4. Lamines, sénescence et vieillissement prématuré. A. Maturation de la lamine A et production de la progérine (mutation des cellules HGPS). La pré-lamine A codée par le gène LMNA va subir une série de modifications post-traductionnelles (carboxyméthylation, farnésylation et clivage) pour devenir la lamine A mature. Dans le syndrome HGPS, une mutation va aboutir à l'usage d'un site d'épissage alternatif, menant à la délétion de 50 acides aminés dans la région du clivage par l'endoprotéase ZMPSTE24. Cette forme mutée de la lamine A ne peut pas être clivée et reste farnésylée. C'est l'accumulation au niveau de l'enveloppe nucléaire de cette forme mutée, non mature et farnésylée de la lamine A qui est responsable de la maladie Progéria. B. Un modèle décrivant le potentiel rôle des lamines dans l'inflammation au cours de la sénescence. Différentes altérations des lamines (par mutations, comme dans le cas de la Progéria, ou une altération du niveau de protéines) aboutissent à des défauts de réparation de l'ADN, à un stress réplicatif, à des altérations télomériques ou encore à des défauts dans le contrôle du stress oxydant. Ces altérations peuvent être associées à l'apparition de lésions de l'ADN, à l'activation de la réponse aux dommages de l'ADN, à l'induction de la sénescence et ainsi à l'induction d'une inflammation liée au SASP.

les fourches de réplication bloquées et sa déplétion empêche leur redémarrage [47]. Dans le cas des cellules HGPS, l'accumulation de progérine induit un effondrement des fourches de réplication en séquestrant le facteur de la réplication, PCNA [48]. II a été également proposé que le stress réplicatif induit par la progérine était associé à une diminution de RAD51, 
nécessaire à la protection et au redémarrage des fourches de réplication. Le stress réplicatif mène à la production de cytokines inflammatoires et d'interférons via la voie cGAS-STING [49]. Des modèles murins d'accumulation de la prélamine $A$ farnésylée présentent aussi une inflammation accrue dépendante de NF- $\kappa B$ [50]. L'accumulation de lamine A immature farnésylée au cours du vieillissement physiologique pourrait donc peut-être contribuer aux pathologies inflammatoires liées à l’âge.

Lamine B1 et sénescence : altération des télomères, stress oxydant et défaut de réparation des dommages de l'ADN

Freund et al. ont montré que la sénescence réplicative ou induite par l'expression d'oncogènes ou l'irradiation à forte dose (10 Gy) est associée à une diminution de la lamine Bl [51]. Shimi et al. ont, quant à eux, montré que la diminution de la lamine Bl induit une sénescence dépendante de $p 53$ [52]. Une diminution de lamine Bl a été observée après induction de l'autophagie, et l'inhibition de cette autophagie atténuerait I'OIS [53]. Ceci contribuerait aux liens existant entre autophagie et sénescence. Notre équipe a mis en évidence que des cellules de patients atteints d'ataxie télangiectasie (AT) (mutées dans le gène $A T M$ de signalisation des $C D B$ ) présentaient une déformation de l'enveloppe nucléaire et un niveau élevé de lamine Bl. L'accumulation de cette protéine est en partie responsable de la sénescence prématurée des cellules AT en culture. Dans ces cellules, le niveau élevé de ROS et l'activation de la p38 MAPK sont responsables de l'accumulation de la lamine B1 [54]. L'équipe de Dreesen a confirmé qu'un niveau élevé de lamine $B l$ induit un arrêt de prolifération (encore plus sévère si la surexpression de la lamine Bl est associée à un défaut de la lamine A) et une sénescence prématurée. L'expression de la télomérase hTERT dans les cellules surexprimant la lamine Bl restaure partiellement la prolifération, suggérant que la surexpression de la lamine Bl induit des altérations télomériques [55]. Ces altérations, dont la cause moléculaire n'est pas encore élucidée, pourraient contribuer à l'établissement de la sénescence observée. Bien que l'induction de sénescence par l'extinction de la lamine Bl soit désormais confirmée par différentes équipes, dont la nôtre, les travaux de l'équipe de Dreesen suggèrent que la diminution de la lamine Bl induirait la sénescence seulement dans des conditions de stress cellulaire (telles qu'une faible densité cellulaire).

L'ensemble de ces données suggèrent donc que la dérégulation des lamines est un élément clé du contrôle de la sénescence. Le ratio lamine Bl/lamine A ainsi que l'intégrité de la structure de l'enveloppe et de la matrice nucléaire pourraient être importants dans ce contrôle. Aujourd'hui, la dérégulation de la lamine Bl est considérée comme une des caractéristiques du phénotype sénescent aux côtés d'un signal persistant de réponse aux dommages de l'ADN, d'une augmentation d'expression des inhibiteurs de cyclines $\mathrm{p} 21^{\mathrm{Cipl}} / \mathrm{pl} 6^{\text {INK4a }}$ et de l'augmentation de sécrétion des facteurs associés à la sénescence (SASP), entre autres. Il est important de noter ici que la seule diminution de la lamine Bl n'est pas spécifique de la sénescence. En effet, elle peut être également associée à la mort cellulaire par apoptose, la lamine Bl étant aussi une cible des caspases.
Certains auteurs s'interrogent pour savoir s'il convient de considérer les perturbations de la lamine Bl comme une cause ou une conséquence de la sénescence. L'analyse des domaines d'ADN associés aux lamines (LAD) a permis de démontrer que la dérégulation de la lamine $\mathrm{Bl}$ dans les cellules sénescentes est associée à des modifications de la chromatine, dont la marque d'histone répressive H3K27me3, liée au contrôle de l'expression des gènes du SASP [56]. La lamine Bl semble être aussi impliquée dans la réponse au stress oxydant, via notamment son interaction avec le facteur de transcription ubiquitaire 0CTl [57]. La déplétion de la lamine Bl pourrait en effet déréguler l'expression des gènes de détoxification des ROS sous contrôle d'OCTl et modifier ainsi la réponse au stress oxydant. II en résulte que les cellules déficientes en lamine $B l$ sont plus sensibles à ces stress oxydants. En accord avec ces données, notre équipe a proposé que la surexpression transitoire de la lamine Bl fait partie de la réponse endogène au stress oxydant, de façon dépendante de l'activation de la p38 MAPK [54]. Une relocalisation d'OCT 1 vers l'enveloppe nucléaire [58] ainsi qu'une résistance accrue à un stress oxydant induit [54] ont d'ailleurs été observées en condition de surexpression de la lamine Bl. Différents travaux suggèrent, de plus, qu'une dérégulation de la lamine Bl aboutit également à des défauts de réparation de l'ADN, et ainsi, potentiellement, à une instabilité génétique ou à une persistance de dommages. ButinIsraeli et al. ont montré que la diminution de la lamine Bl induit une dérégulation de l'expression de différents gènes impliqués dans la réparation des CDB [59]. La lamine $B l$ participerait aussi à la stabilité de la protéine RAD51, impliquée dans la réparation des CDB par recombinaison homologue. Une interaction entre la lamine Bl et la protéine RAD51 a été décrite [60]. Un défaut de réparation par excision de nucléotide (NER), impliquée dans la prise en charge des dommages induits par les rayons ultra-violets ou par des agents induisant des pontages de I'ADN, a aussi été rapporté [61]. Cependant, la conséquence de ces altérations, ayant pour origine la diminution de l'expression de la lamine Bl, sur l'induction potentielle de la sénescence n'est toutefois pas clairement élucidée. Enfin, l'induction de la voie cGAS par la présence d'ADN dans le cytosol semble être associée, dans différentes conditions, à la présence de micro-noyaux. Le niveau de la lamine Bl ou de la lamine B2 participe à la stabilité de l'enveloppe des micronoyaux et pourrait ainsi indirectement réguler l'activation de cGAS, de NF- $\kappa B$, du SASP et l'inflammation.

Les perturbations de l'architecture nucléaire et du réseau de lamines pourraient donc activer des signaux capables de faire rentrer les cellules en sénescence, 
en altérant leur cycle cellulaire, leur potentiel rédox (oxydo-réduction), et potentiellement, leur stabilité télomérique, ou plus généralement chromosomique, ainsi que leur environnement inflammatoire (Figure 4B). Elles participeraient ainsi au vieillissement de l'organisme.

\section{Conclusions}

Nous avons vu que différentes situations de sénescence ou de vieillissement accéléré ont en commun l'activation de la réponse aux dommages de I'ADN, associée ou non à la persistance de dommages télomériques, le stress oxydant, des perturbations de l'enveloppe nucléaire, en particulier une altération des lamines, ainsi que l'induction d'un phénotype inflammatoire. L'ensemble de ces facettes de la sénescence, que l'on pensait potentiellement indépendantes sont en fait toutes associées les unes aux autres. Des recherches fondamentales pour mieux comprendre les mécanismes d'induction de la sénescence sont nécessaires. II est peu probable que l'on puisse s'opposer un jour totalement au vieillissement au niveau de l'organisme, mais il n'est pas invraisemblable, à l'avenir, de vivre beaucoup plus âgé en meilleure, voire en très bonne santé. De telles recherches permettront de proposer des traitements pour certaines maladies de vieillissement accéléré, telles que la Progéria, et offriront aussi la possibilité de prévenir ou de soulager les différentes maladies liées à l'âge. Cela représente un enjeu sociétal et économique majeur. La découverte, entre autres, de nouveaux sénolytiques permettant d'éliminer les cellules sénescentes, ou de nouveaux modulateurs du SASP, constituent un grand espoir. Toutefois, il convient de ne pas oublier la théorie de la pléiotropie antagoniste et l'action anti-tumorale de la sénescence et du SASP, afin de gérer les potentiels effets indésirables de ce genre d'approches thérapeutiques. Des recherches actives sont donc capitales dans le domaine du vieillissement, mais toujours en lien également avec celui de l'oncologie. $\diamond$

\section{SUMMARY}

Aging : A matter of DNA damage, nuclear envelope alterations and inflammation?

The accumulation of senescent cells accompanies organismal aging. Senescent cells produce an inflammatory microenvironment that is conducive to the development of many age-related diseases. Here we describe the different situations leading to cellular senescence and show that these situations are frequently associated with DNA damage. We also discuss the intimate link between cell aging and perturbations in the nuclear envelope, namely in nuclear lamins, as seen in progeroid syndromes. Finally, we present evidence that these alterations are associated with DNA repair defects, the persistence of DNA damage, and an inflammatory phenotype. $\diamond$

\section{REMERCIEMENTS}

Nous tenons à remercier l'ensemble de l'équipe LREV, en particulier Dr Emilie Rass pour ses commentaires sur cette synthèse. L'équipe est soutenue par l'Inserm, le comité régional Île-de-France de la Ligue contre le Cancer, AT Europe, le Cancéropôle IDF (AAP Emergence) I'INCA, l'EDF et le programme Radiobiologie du CEA. PF est financée par l'Université Paris Saclay et la Ligue Nationale contre le Cancer et SW est financé par un financement de Thèse $C E A D R F$ «Phare » et la Fondation ARC. AM a été financée par un financement de thèse CEA Irtelis et la Fondation ARC. Les Figures ont été créés avec Biorender.com.
LIENS D'INTÉRÊT

Les auteurs déclarent n'avoir aucun lien d'intérêt concernant les données publiées dans cet article.

\section{RÉFÉRENCES}

1. Hernandez-Segura A, Nehme J, Demaria M. Hallmarks of cellular senescence. Trends Cell Biol $2018 ; 28:$ 436-53.

2. Rodier F, Munoz D, Teachenor R, et al. DNA-SCARS: distinct nuclear structures that sustain damage-induced senescence growth arrest and inflammatory cytokine secretion. J Cell Sci 2011 ; 124 : 68-81.

3. Brondello JM, Prieur A, Philipot D, et al. La sénescence cellulaire : un nouveau mythe de Janus? Med Sci (Paris) $2012 ; 28: 288-96$.

4. Childs BG, Baker DJ, Kirkland JL, et al. Senescence and apoptosis: dueling or complementary cell fates? EMBO Rep 2014 ; 15 : 1139-53.

5. Coppé JP, Desprez Py, Krtolica A, et al. The senescence-associated secretory phenotype: the dark side of tumor suppression. Annu Rev Pathol $2010 ; 5$ : 99-118.

6. Ito Y, Hoare M, Narita M. Spatial and temporal control of senescence. Trends Cell Biol $2017 ; 27: 820-32$.

7. WIIliams GC. Pleiotropy, natural selection, and the evolution of senescence. Evolution 1957 ; 398

8. Rodier F, Campisi J. Four faces of cellular senescence. J Cell Biol 2011 ; 192 : 547-56.

9. Baker D, Wijshake T, Tchkonia T, et al. Clearance of pl6Ink4a-positive senescent cells delays ageing-associated disorders. Nature 2011 ; 479 : 232-6.

10. Paez-Ribes M, González-Gualda $\varepsilon$, Doherty GJ, et al. Targeting senescent cells in translational medicine. EMBO Mol Med 2019; 11 : e10234.

11. Ovadya $Y$, Landsberger $T$, Leins $H$, et al. Impaired immune surveillance accelerates accumulation of senescent cells and aging. Nat Commun 2018 ; 9.

12. Perez-Lanzon $M$, Zitvogel L, Kroemer G. Failure of immunosurveillance accelerates aging. Oncoimmunology 2019; 8 : el575117.

13. Serrano M, Lin AW, McCurrach ME, et al. Oncogenic ras provokes premature cell senescence associated with accumulation of p53 and pl6INK4a. Cell $1997 ; 88: 593-602$.

14. Zhu H, Blake S, Kusuma FK, et al. Oncogene-induced senescence: from biology to therapy. Mech Ageing Dev 2020 ; 187 : 111229.

15. Bartkova J, Rezaei N, Liontos M, et al. Oncogene-induced senescence is part of the tumorigenesis barrier imposed by DNA damage checkpoints. Nature $2006 ; 444: 633-7$.

16. Kuilman T, Michaloglou C, Mooi WJ, et al. The essence of senescence. Genes Dev $2010 ; 24: 2463-79$.

17. Bianchi-Smiraglia A, Nikiforov MA. Controversial aspects of oncogeneinduced senescence. Cell Cycle $2012 ; 11: 4147-51$.

18. Jeanblanc M, Ragu S, Gey C, et al. Parallel pathways in RAF-induced senescence and conditions for its reversion. Oncogene $2012 ; 31: 3072-85$.

19. Michaloglou C, Vredeveld LCW, Mooi WJ, et al. BRAF $\varepsilon 600$ in benign and malignant human tumours. Oncogene 2008 ; 27 : 877-95.

20. Astle MV, Hannan KM, Ng PY, et al. AKT induces senescence in human cells via $\mathrm{mTORCl}$ and $\mathrm{p} 53$ in the absence of DNA damage: implications for targeting mTOR during malignancy. Oncogene 2012 ; 31 : 1949-62.

21. Wiley CD, Velarde MC, Lecot $P$, et al. Mitochondrial dysfunction induces senescence with a distinct secretory phenotype. Cell Metabol 2016; 23 : 303-14.

22. Freund A, Patil CK, Campisi J. p38MAPK is a novel DNA damage responseindependent regulator of the senescence-associated secretory phenotype. EMBO J 2011 ; 30 : 1536-48.

23. Hiebert $P$, Wietecha MS, Cangkrama M, et al. Nrf2-mediated fibroblast reprogramming drives cellular senescence by targeting the matrisome. Developmental Cell 2018 ; 46 : 145-61.el0.

24. Campisi J. Aging, cellular senescence, and cancer. Annu Rev Physiol 2013; $75: 685-705$

25. Acosta JC, Banito A, Wuestefeld T, et al. A complex secretory program orchestrated by the inflammasome controls paracrine senescence. Nat Cell Biol 2013 ; 15 : 978-90.

26. Rodier F, Coppe J, Patil C, et al. Persistent DNA damage signalling triggers senescence-associated inflammatory cytokine secretion. Nat Cell Biol 2009 ; $11: 973-9$

27. Härtlova A, Erttmann SF, Raffi FA, et al. DNA damage primes the type I interferon system via the cytosolic DNA sensor sting to promote antimicrobial innate immunity. Immunity $2015 ; 42$ : 332-43. 


\section{RÉFÉRENCES}

28. Coquel F, Neumayer C, Lin YL, et al. SAMHDI and the innate immune response to cytosolic DNA during DNA replication. Curr Opin Immunol $2019 ; 56: 24-30$.

29. Chanut R, Petrilli V. Détection de l'ADN cytosolique par la voie cGAS-STING : de l'immunité innée vers le contrôle de la tumorigenèse. Med Sci (Paris) 2019 ; $35: 527-34$

30. Dou Z, Ghosh K, Vizioli MG, et al. Cytoplasmic chromatin triggers inflammation in senescence and cancer. Nature $2017 ; 550: 402-6$.

31. Glück S, Guey B, Gulen MF, et al. Innate immune sensing of cytosolic chromatin fragments through cGAS promotes senescence. Nat Cell Biol $2017 ; 19: 1061-70$.

32. Yang $\mathrm{H}$, Wang $\mathrm{H}$, Ren J, et al. cGAS is essential for cellular senescence. Proc Natl Acad Sci USA 2017 ; 114 : ع4612-20.

33. De Cecco M, Ito T, Petrashen AP, et al. Ll drives IFN in senescent cells and promotes ageassociated inflammation. Nature $2019 ; 566: 73-8$.

34. Eriksson M, Brown WT, Gordon LB, et al. Recurrent de novo point mutations in lamin A cause Hutchinson-Gilford progeria syndrome. Nature $2003 ; 423$ : 293-8.

35. De Sandre-Giovannoli A, Bernard R, Cau P, et al. Lamin a truncation in Hutchinson-Gilford progeria. Science $2003 ; 300: 2055$

36. Nissan X, Blondel S, Navarro C, et al. Unique preservation of neural cells in Hutchinson-Gilford progeria syndrome is due to the expression of the neural-specific miR-9 microRNA. Cell Rep 2012 ; 2:1-9.

37. Scaffidi P, Misteli T. Lamin A-dependent nuclear defects in human aging. Science 2006 ; 312 : 1059-63.

38. Redwood AB, Perkins SM, Vanderwaal RP, et al. A dual role for A-type lamins in DNA double-strand break repair. Cell Cycle $2011 ; 10: 2549-60$.

39. Cobb AM, Larrieu D, Warren DT, et al. Prelamin A impairs 53BPl nuclear entry by mislocalizing NUP153 and disrupting the Ran gradient. Aging Cell 2016; 15 : 1039-50.

40. Liu B, Wang J, Chan KM, et al. Genomic instability in laminopathy-based premature aging. Nat Med $2005 ; 11: 780-5$.

41. Burla R, La Torre M, Saggio I. Mammalian telomeres and their partnership with lamins. Nucleus $2016 ; 7: 187-202$

42. Wood AM, Rendtlew Danielsen JM, Lucas CA, et al. TRF2 and lamin A/C interact to facilitate the functional organization of chromosome ends. Nat Commun $2014 ; 5: 5467$.

43. Aguado J, Sola-Carvajal A, Cancila V, et al. Inhibition of DNA damage response at telomeres improves the detrimental phenotypes of Hutchinson-Gilford progeria syndrome. Nat Commun $2019 ; 10: 4990$

44. Richards S, Muter J, Ritchie P, et al. The accumulation of un-repairable DNA damage in laminopathy progeria fibroblasts is caused by ROS generation and is prevented by treatment with $\mathrm{N}$-acetyl cysteine. Hum Mol Genet $2011 ; 20: 3997-4004$.

45. Kubben $\mathrm{N}$, Zhang $\mathrm{W}$, Wang $\mathrm{L}$, et al. Repression of the antioxidant NRF2 pathway in premature aging. Cell $2016 ; 165: 1361-74$.

46. Fabrini R, Bocedi A, Pallottini V, et al. Nuclear Shield: a multi-enzyme task-force for nucleus protection. PloS One 2010 ; 5 : el4125.
47. Singh $M$, Hunt $C R$, Pandita RK, et al. Lamin A/C depletion enhances dna damageinduced stalled replication fork arrest. Mol Cell Biol 2013; 33 : 1210-22.

48. Hilton BA, Liu J, Cartwright BM, et al. Progerin sequestration of PCNA promotes replication fork collapse and mislocalization of XPA in laminopathy-related progeroid syndromes. FASEB J 2017 ; 31 : 3882-93.

49. Coll-Bonfill N, Cancado de Faria R, Bhoopatiraju S, et al. Calcitriol prevents RAD51 loss and cGAS-STING-IFN response triggered by progerin. Proteomics 2019 ; el800406.

50. Osorio F, Barcena C, Soria-Valles C, et al. Nuclear lamina defects cause ATMdependent NF-kappaB activation and link accelerated aging to a systemic inflammatory response. Genes Dev 2012; $26: 2311-24$.

51. Freund A, Laberge R, Demaria M, et al. Lamin Bl loss is a senescenceassociated biomarker. Mol Biol Cell 2012; 23 : 2066-75.

52. Shimi T, Butin-Israeli V, Adam S, et al. The role of nuclear lamin Bl in cell proliferation and senescence. Genes Dev $2011 ; 25: 2579-93$.

53. Dou Z, Xu C, Donahue G, et al. Autophagy mediates degradation of nuclear lamina. Nature $2015 ; 527$ : 105-9.

54. Barascu A, Le Chalony C, Pennarun G, et al. Oxidative stress induces an ATM independent senescence pathway through p38 MAPK-mediated lamin BI accumulation. EMBO J $2012 ; 31: 1080-94$

55. Dreesen 0 , Chojnowski A, Ong P, et al. Lamin Bl fluctuations have differential effects on cellular proliferation and senescence. J Cell Biol $2013 ; 200: 605-17$.

56. Shah PP, Donahue G, Otte GL, et al. Lamin Bl depletion in senescent cells triggers large-scale changes in gene expression and the chromatin landscape. Genes Dev 2013; $27: 1787-99$

57. Malhas AN, Lee CF, Vaux DJ. Lamin Bl controls oxidative stress responses via Oct-1.J Cell Biol $2009 ; 184: 45-55$.

58. Columbaro M, Mattioli $\varepsilon$, Maraldi NM, et al. Oct-1 recruitment to the nuclear envelope in adult-onset autosomal dominant leukodystrophy. Biochim Biophys Acta $2013 ; 1832$ : 411-20.

59. Butin-Israeli V, Adam S, Jain N, et al. Role of lamin bl in chromatin instability. Mol Cell Biol 2015 ; 35 : 884-98.

60. Liu N, Sun J, Kono K, et al. Regulation of homologous recombinational repair by lamin $B 1$ in radiation-induced DNA damage. FASEB J $2015 ; 29$ : 2514-25.

61. Butin-Israeli V, Adam SA, Goldman RD. Regulation of nucleotide excision repair by nuclear lamin bl. PLoS One 2013; 8 : e69169.

62. Vermeij WP, Hoeijmakers JHJ, Pothof J. Genome integrity in aging: human syndromes, mouse models, and therapeutic options. Annu Rev Pharmacol Toxicol $2016 ; 56: 427-45$

TIRÉS À PART

P. Bertrand

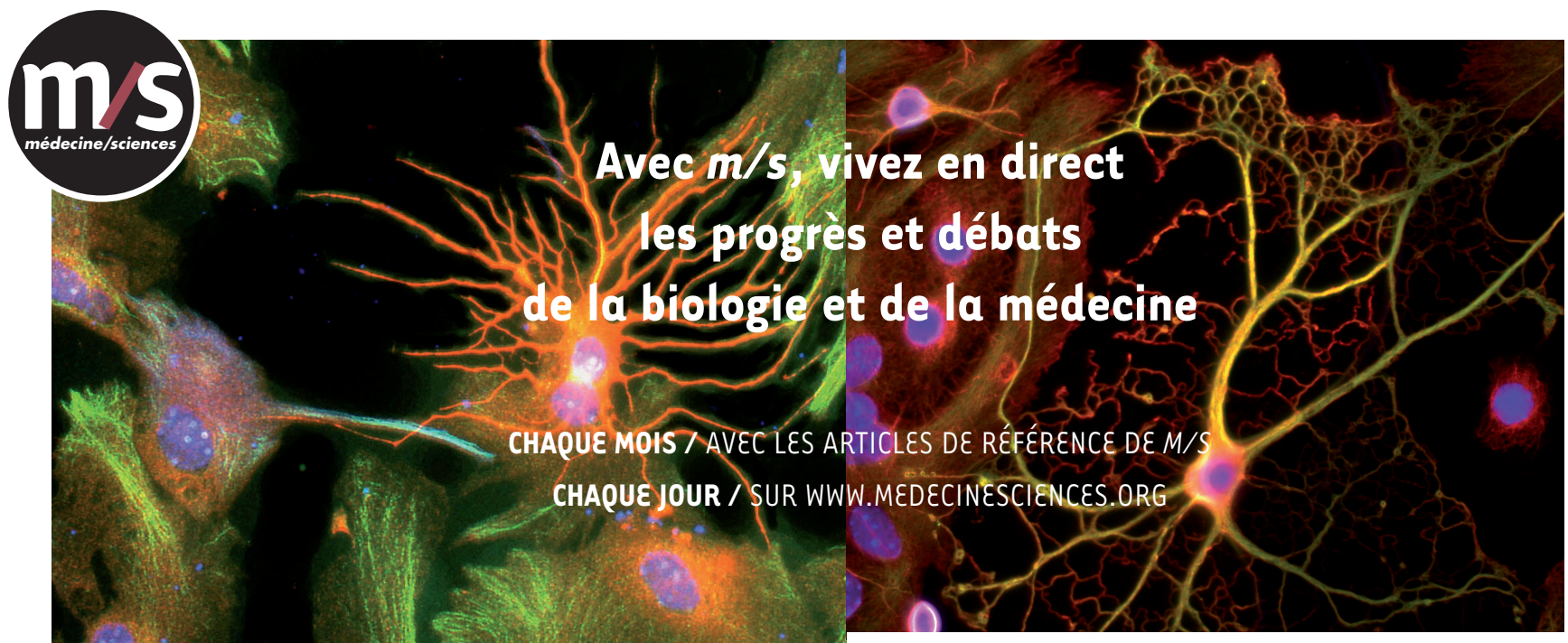

Abonnez-vous sur

wWw.medecinesciences.org 